CP3-12-XX

\title{
Effective field theory approach to the Higgs lineshape
}

\author{
Diogo Buarque Franzosi, Fabio Maltoni, Cen Zhang \\ Centre for Cosmology, Particle Physics and Phenomenology, \\ Université Catholique de Louvain, B-1348 Louvain-la-Neuve, Belgium
}

\begin{abstract}
The phenomenology of unstable particles, including searches and exclusion limits at the LHC, depends significantly on its lineshape. When the width of the resonance is large with respect to its mass, off-shell effects become relevant and the very same definition of width becomes non trivial. Taking a heavy Higgs boson as an example, we propose a new formulation to describe the lineshape via an effective field theory approach. Our method leads to amplitudes that are gauge invariant, respect unitarity and can appropriately describe the lineshape of broad resonances. The application of the method to the following relevant processes for the LHC phenomenology have been considered: gluon fusion, vector boson scattering and $t \bar{t}$ production via weak boson fusion.

PACS numbers: 14.80
\end{abstract}

\section{INTRODUCTION}

The CMS and ATLAS collaborations have announced the discovery of a resonance around $126 \mathrm{GeV}$ compatible with the Higgs boson predicted by the Standard Model (SM) [1, 2]. To eventually confirm the discovery of the SM Higgs boson, it will be necessary not only to measure the strength and the structure of its couplings to the fermions and vector bosons of the SM, but also to exclude the existence of other heavier scalar states with similar properties.

The scalar sector of the SM is particularly simple, yet it does not provide any clue either on its origin, i.e. on the possible underlying dynamics, or on its stability. Alternative models that address these and other open questions often predict a richer structure for the scalar sector, such as in supersymmetry (SUSY), Technicolor theories and models with extra dimensions. Two-Higgs doublet models provide the simplest and most often studied extensions of the scalar sector of the SM. In all these cases, heavier scalar and/or pseudo scalar partners of the "SM Higgs" can be expected below the TeV scale.

The width of such heavy states, turns out to be sizable. A large width induces both a smearing and deformation of the signal line shape as well as a sizable signal/background interference. For a heavy SM-like Higgs, (e.g, $\Gamma \simeq 100 \mathrm{GeV}$ for $m_{H} \simeq 550 \mathrm{GeV}$ ), the narrow width approximation (NWA) has been shown to be untenable, possibly invalidating the currently set exclusion limits on the heavy Higgs and beyond [3, 4] .

Going beyond the NWA, however, turns out not to be such an easy theoretical task. The challenges are manifold. First, the most accurate predictions for the signal cross sections, typically at the next-to-next-to-leading order (NNLO) in QCD and at NLO in EW , assume a complete factorization between production and decay, i.e., they employ the NWA, and the a posteriori inclusion of width effects is not uniquely defined [5]. Second, the very definition of a width, which amounts to a resummation of a specific subset of terms appearing at all orders in perturbation theory becomes problematic, leading to possible violations of gauge symmetry as well as of unitarity [6].

Currently, the most pragmatic and employed solution is the so-called complex mass scheme (CMS) [7]. In short, it amounts to analytically continue the parameters entering the SM Lagrangian that are related to the masses to complex values. Such scheme maintains gauge invariance at all orders in perturbation theory and can be consistently employed in (N)NLO in EW computations [8, 9]. However, since a fixed complex pole is assumed for any virtuality of the unstable particle, the resulting scattering amplitudes violate unitarity and do not correctly describe the lineshape of broad resonances.

An alternative to the CMS is the fermion-loop scheme [6, 10], which offers a solution for the restoration of unitarity, but yet does not give a complete description of unstable particles because the width of a heavy Higgs is dominated by its decay into gauge bosons. A consistent treatment of the bosonic contributions is possible in the framework of the background field gauge 11], but this requires a calculation of the complete radiative corrections at a fixed loop order. These two approaches require greatly increase the complexity of the calculation. Other suggested schemes for the treatment of unstable particles include the pole scheme [12], the Seymour scheme [13], the use of an effective Lagrangian including non-local interactions 14, 15], and an approach based on collinear effective field theory [16].

Summarizing, in general and especially for a heavy Higgs, one would like to be able to include a running width in the propagator directly connected with the Higgs self-energy and at the same time to respect gauge invariance and unitarity.

In this work we tackle the problem from an effective field theory (EFT) point of view. We propose to system- 
atically include width effects via a set of gauge invariant higher dimensional terms to the SM lagrangian, along the lines of what was first proposed in Ref. [14, 15]. Such new operators systematically encapsulate higher order terms coming from the self-energy and naturally allow a running and physical width for the Higgs in a gauge invariant way. As we will show in the following, our scheme is consistent at higher orders and it can be considered a generalization of the CMS as it reduces to it in the limit where the dependence on the virtuality of the Higgs selfenergy is neglected.

\section{SETTING UP THE STAGE}

The two-point Green's function for the Higgs boson is

$$
\Delta_{H}(s)=s-m_{H, 0}^{2}+\Pi_{H H}(s),
$$

where $m_{H, 0}$ is the bare mass, and $\Pi_{H H}(s)$ is the Higgs self-energy. In the conventional on-shell definition, the mass and width are given by

$$
\begin{aligned}
& m_{H, O S}^{2}=m_{H, 0}^{2}-\operatorname{Re} \Pi\left(m_{H, O S}^{2}\right), \\
& m_{H, O S} \Gamma_{H, O S}=\frac{\operatorname{Im} \Pi\left(m_{H, O S}^{2}\right)}{1+\operatorname{Re} \Pi^{\prime}\left(m_{H, O S}^{2}\right)} .
\end{aligned}
$$

These definitions become gauge-dependent at order $\mathcal{O}\left(g^{4}\right)$.

In order to avoid the divergence of the tree-level propagator $D(s)=i /\left(s-m_{H, O S}^{2}\right)$, one performs the Dyson resummation to obtain

$$
D(s)=\frac{i}{s-m_{H, O S}^{2}+i m_{H, O S} \Gamma_{H, O S}} .
$$

To include the running effects of the width, one can further approximate the propagator by

$$
D(s)=\frac{i}{s-m_{H, O S}^{2}+i \operatorname{Im} \Pi(s)},
$$

where the imaginary part of $\Pi(s)$ is related to the Higgsboson width. The consistency of the above treatments of the Higgs propagator with the equivalence theorem and unitarity has been discussed by Valencia and Willenbrock [17.

Alternatively, as shown in a series of papers [12, 18] a consistent, convenient and resilient definition of mass $\mu$ and width $\gamma$ up to two loops, is obtained by setting $s_{H} \equiv \mu^{2}-i \mu \gamma$ and then solving the implicit equation

$$
s_{H}-m_{H, 0}^{2}+\Pi_{H H}\left(s_{H}\right)=0
$$

in terms of $s_{H}$. This gives a gauge independent definition to all orders [19] (independent of the gauge choice present in the computation of $\left.\Pi_{H H}\left(s_{H}\right)\right)$ and in addition avoids unphysical threshold singularities [20].

The above definition is also consistent with the use of the CMS. In this scheme the propagator is $\Delta_{H}^{-1}(s)=$ $s-s_{H}$. By definition this approach can give a good approximation of the full propagator

$$
\Delta_{H}^{-1}(s)=\frac{1}{s-s_{H}+\Pi_{H H}^{R}(s)}
$$

only close to the pole or, equivalently, for a small width, $\gamma / \mu \ll 1$. Here $\Pi_{H H}^{R}(s)$ is the renormalized self energy, satisfying the following renormalization conditions:

$$
\Pi_{H H}^{R}\left(s_{H}\right)=0, \quad \Pi_{H H}^{R}\left(s_{H}\right)=0 .
$$

A natural improvement would consist in including the full resummed propagator in explicit calculations. This, however, leads to gauge violation already at the tree level. The reason being that in perturbation theory gauge invariance is guaranteed order by order while the presence of a width implies the resummation of a specific subset of higher order contributions, the self-energy corrections. This results in a mixing of different orders of perturbation theory. In particular, the following issues need to be addressed:

1. In general $\Pi_{H H}(s)$ explicitly depends on the gaugefixing parameter (GFP). To resum the self-energy correction to all orders, $\Pi_{H H}(s)$ must be extracted in a physically meaningful way.

2. The resummed propagator spoils the gauge cancellation among different diagrams, and eventually leads to the violation of Goldstone-boson equivalence theorem and unitarity bound.

Both issues can be tackled by the so-called Pinch Technique (PT) 21 24]. In the PT framework, a modified one-loop self-energy for the Higgs boson can be constructed by appending to the conventional self energy additional propagator-like contributions concealed inside vertices and boxes. For the application of $\mathrm{PT}$ in resonant transition amplitude, and in particular, the extraction of a physical self energy, we refer to the work of Refs. 2529].

The modified self-energy correction for the Higgs is GFP-independent, and reflects properties generally associated with physical observables. At the one loop level, we have the following expressions [28, 30] 


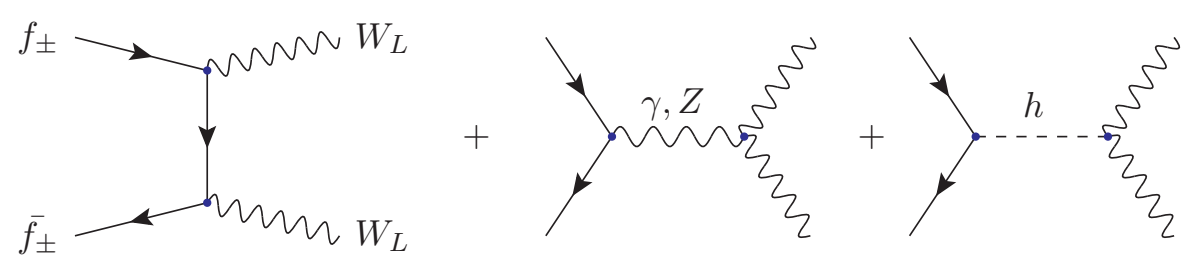

FIG. 1: Diagrams contributing to fermion anti-fermion scattering into longitudinal $W$ 's.

$$
\begin{aligned}
\Pi_{H H}^{(W W)}(s) & =\frac{\alpha_{W}}{16 \pi} \frac{m_{H}^{4}}{m_{W}^{2}}\left[1+4 \frac{m_{W}^{2}}{m_{H}^{2}}-4 \frac{m_{W}^{2}}{m_{H}^{4}}\left(2 s-3 m_{W}^{2}\right)\right] B_{0}\left(s, m_{W}^{2}, m_{W}^{2}\right), \\
\Pi_{H H}^{(Z Z)}(s) & =\frac{\alpha_{W}}{32 \pi} \frac{m_{H}^{4}}{m_{W}^{2}}\left[1+4 \frac{m_{Z}^{2}}{m_{H}^{2}}-4 \frac{m_{Z}^{2}}{m_{H}^{4}}\left(2 s-3 m_{Z}^{2}\right)\right] B_{0}\left(s, m_{Z}^{2}, m_{Z}^{2}\right) \\
\Pi_{H H}^{(f f)}(s) & =\frac{3 \alpha_{W}}{8 \pi} \frac{m_{f}^{2}}{m_{W}^{2}}\left(s-4 m_{f}^{2}\right) B_{0}\left(s, m_{f}^{2}, m_{f}^{2}\right) \\
\Pi_{H H}^{(H H)}(s) & =\frac{9 \alpha_{W}}{32 \pi} \frac{m_{H}^{4}}{m_{W}^{2}} B_{0}\left(s, m_{H}^{2}, m_{H}^{2}\right)
\end{aligned}
$$

where the superscripts denote the contributions from the $W, Z$, fermions and Higgs loops, and

$$
\begin{aligned}
& B_{0}\left(p^{2}, m_{1}^{2}, m_{2}^{2}\right) \\
& \quad \equiv(2 \pi \mu)^{4-d} \int \frac{d^{d} k}{i \pi^{2}} \frac{1}{\left(k^{2}-m_{1}^{2}\right)\left[(k+p)^{2}-m_{2}^{2}\right]}
\end{aligned}
$$

is the normal Passarino-Veltman function [31]. These results are indepent of the GFP. Note that the expressions in Eqs. (9) 12) coincide with the $\xi=1$ result obtained in the background-field gauge 32 34].

In addition, the gauge cancellation among different amplitudes can be restored, by including certain vertex corrections obtained via the PT [30, 35]. This is because in this framework the Green's functions satisfy the tree-level-like Ward identities (WI), which are crucial for ensuring the gauge invariance of the resummed amplitude.

As an example, let us consider the Higgs-mediated part of same helicity fermion scattering into longitudinal $W^{\prime}$ 's, $f_{ \pm} \bar{f}_{ \pm} \rightarrow W_{L}^{+} W_{L}^{-}$. There are contributions from $s-$ channel and $t$-channel diagrams, as is shown in Figure 1. The contributions from $t$-channel and Higgs diagram to the amplitudes coming from longitudinal components of the $W$ 's and same helicity fermions (in the high energy limit) read ${ }^{1}$

$$
\begin{aligned}
\mathcal{M}_{h}^{L} & \equiv \mathcal{M}_{s}^{\mu \nu} \frac{k_{1 \mu} k_{2 \nu}}{m_{W}^{2}} \\
& =\frac{-i g m_{f}}{2 m_{W}} \bar{v}\left(p_{2}\right) u\left(p_{1}\right) \frac{i}{\Delta_{H}(s)} \Gamma^{H W W, \mu \nu}\left(q, k_{1}, k_{2}\right) \frac{k_{1 \mu} k_{2 \nu}}{m_{W}^{2}} \\
\mathcal{M}_{t}^{L} & \equiv \mathcal{M}_{t, Z}^{\mu \nu} \frac{k_{1 \mu} k_{2 \nu}}{m_{W}^{2}} \\
& =-\frac{i g^{2} m_{f}}{4 m_{W}^{2}} \bar{v}\left(p_{2}\right) u\left(p_{1}\right)+\cdots
\end{aligned}
$$

where $\Gamma_{\mu \nu}^{H W W}\left(q, k_{1}, k_{2}\right)$ is the $H W^{+} W^{-}$vertex. The ellipsis in $\mathcal{M}_{t}^{L}$ denotes terms that are not related to the Higgs exchange diagram. These terms come from the contribution of opposite helicity fermions, and are supposed to cancel the bad high-energy behavior of the $\gamma / Z$ mediated diagrams.

Without the Higgs contribution $\mathcal{M}^{L}$ grows with energy and eventually violates unitarity. The cancellation of the bad high-energy behavior of each amplitude, and the equivalence theorem, are guaranteed by the following WI:

$$
\begin{aligned}
& k_{+}^{\mu} k_{-}^{\nu} \Gamma_{\mu \nu}^{H W W}\left(q, k_{+}, k_{-}\right)= \\
& -m_{W}^{2} \Gamma^{H \phi^{+} \phi^{-}}\left(q, k_{+}, k_{-}\right)+\frac{i g m_{W}}{2} \Delta_{H}\left(q^{2}\right),
\end{aligned}
$$

where $\phi^{ \pm}$are Nambu-Goldstone bosons. Only the leading terms at high energy are included. The relation above

\footnotetext{
${ }^{1}$ Here we assume $\epsilon_{1,2}^{\mu} \approx k_{1,2}^{\mu} / m_{W}$ at high energy region.
} 
explicitly shows that the inclusion of higher order terms in the imaginary part of $\Delta_{H}\left(q^{2}\right)$ has to be related to the EW corrections of $\Gamma_{\mu \nu}^{H W W}$ and three scalar vertex. Only if both $\Delta_{H}(s)$ and $\Gamma_{\mu \nu}^{H W W}$ are computed in one-loop via the $\mathrm{PT}$, then the WI remains valid, and the gaugecancellation, as well as the equivalence theorem, are not spoiled. Besides, $\mathcal{M}_{t, Z}^{L}$ is not affected by the Higgs width and therefore the tree-level relations can be used. Thus the resummed propagator can be consistently included with the one-loop correction to $\Gamma_{\mu \nu}^{H W W}$ via the PT.

Even though correct, the solution outlined above for $f \bar{f} \rightarrow W_{L}^{+} W_{L}^{-}$is not a general one. In $W_{L}^{+} W_{L}^{-} \rightarrow Z_{L} Z_{L}$, for example, it is not sufficient to include only the $H W W$ and $H Z Z$ corrections. The triple and quartic vectorboson vertices at one-loop are also required to cancel the bad high-energy behavior of the Higgs-mediated amplitude and the overall procedure of analyzing the full set of WI's becomes more and more involved. The goal of this work is to present a simple method to generate the needed corrections to the vertices and propagators so that the WI's are automatically satisfied and unitarity automatically ensured.

\section{THE EFT APPROACH}

As explained above, we aim at finding a systematic approach to improve the Higgs propagator without breaking either gauge invariance or unitarity. In other words we are looking for a mechanism that guarantees the constraints imposed by the WI to be satisfied at any order in perturbation theory.

At one loop, the full calculation via the PT certainly provides an exact solution valid at NLO. The challenge is to achieve the same keeping the calculation at leading order, including only the necessary ingredients coming from $\mathrm{NLO}$ and resumming them into the propagator via a Dyson-Schwinger approach. The idea is to associate the corrections to an ad hoc constructed gauge-invariant operator and match the operator to the one-loop twopoint function $\Delta_{H}(s)$ calculated via the PT. In so doing one aims at obtaining the exact resummed propagator already at the leading order and, at the same time, the interactions modified to automatically satisfy the WI's. The latter desired result ensures the gauge-invariance of the amplitudes, and it can be considered as an approximation to a full one-loop calculation in PT.

To this aim, we consider the Taylor expansion of the function $\Pi(s)=\Pi_{H H}^{R}(s)$

$$
\Pi(s)=\sum_{i=0}^{\infty} c_{i} s^{i}
$$

where $c_{i}$ are dimensionful constants and, as first attempt, we add the following infinite set of operators to the La- grangian:

$$
\begin{aligned}
\mathcal{O}_{\Pi} & =\sum_{i=0}^{\infty} c_{i} \phi^{\dagger}\left(-D^{2}\right)^{i} \phi \\
& \equiv \phi^{\dagger} \Pi\left(-D^{2}\right) \phi
\end{aligned}
$$

where $\phi$ is the Higgs doublet, and $D^{\mu}$ is the covariant derivative. It is straightforward to check that $\mathcal{O}_{\Pi}$ modifies the Higgs propagator as desired: the two $\phi$ 's contribute two Higgs fields, and each $-D^{2}$ contributes an $s$ leading to

$$
\Pi(s)=\Pi_{H H}^{R}(s),
$$

as desired. Note that in principle, $\mathcal{O}_{\Pi}$ is a non-local operator, yet by expanding it, we re-express it in terms of an infinite series of local operators. ${ }^{2}$

We remark that while very similar in spirit, our approach differs from that of Ref. [14]: the operator chosen there does not contain gauge fields, and it is therefore not sufficient to restore the gauge cancellation and fix the bad high-energy behavior in vector-vector scattering.

Eq. (17) leads to the correct expression for the propagator. However, the first term $\Pi(0) \phi^{\dagger} \phi$ in the expansion corresponds to a tadpole contribution. This can be avoided if this term is replaced by

$$
\Pi(0) \phi^{\dagger} \phi \rightarrow \frac{\Pi(0)}{2 v^{2}}\left[\left(\phi^{\dagger} \phi\right)-\frac{v^{2}}{2}\right]^{2}
$$

i.e., the Higgs-self interaction is suitably modified. As one can easily check, such a modification leaves the relation of Eq. (18) unchanged. The final form of the operator, which we dub $\tilde{\mathcal{O}}_{\Pi}$, is

$$
\tilde{\mathcal{O}}_{\Pi}=\phi^{\dagger}\left[\Pi\left(-D^{2}\right)-\Pi(0)\right] \phi+\frac{\Pi(0)}{2 v^{2}}\left[\left(\phi^{\dagger} \phi\right)-\frac{v^{2}}{2}\right]^{2}
$$

The addition of this operator to the SM leads to several changes, which we now consider in detail. First of all, by construction, it gives rise to the propagator in Eq. (77), and a resummed propagator with the full one-loop self energy via the PT at tree level is obtained. Second it leads to modifications of the other interactions, in such a way that gauge invariance is maintained. For example, the $W$ and $Z$ two-point functions are modified by the

\footnotetext{
${ }^{2}$ In general, inclusion of higher-order derivatives in the Lagrangian leads to very peculiar quantum field theories, aka Lee-Wick theories, see [36] for a recent analysis and references. As we are going to see later, in our approach, we only use the imaginary part of $\Pi(s)$ and therefore the real part of the propagator is not affected.
} 


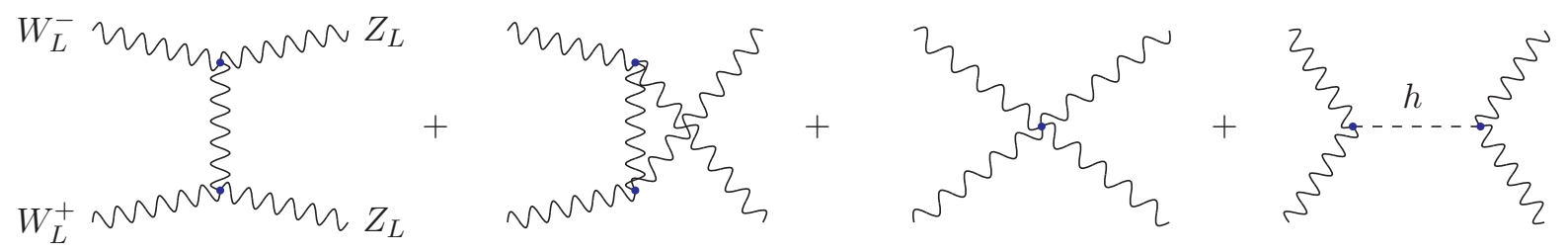

FIG. 2: Diagrams contributing to $W_{L}^{+} W_{L}^{-} \rightarrow Z_{L} Z_{L}$

addition of

$$
\begin{aligned}
i \Delta \Pi_{W W}^{\mu \nu}\left(q^{2}\right) & =i\left(\frac{g v}{2}\right)^{2}\left[\Pi^{\prime}(0) g^{\mu \nu}+\Pi^{\prime \prime}\left(q^{2}\right) q^{\mu} q^{\nu}\right] \\
i \Delta \Pi_{Z Z}^{\mu \nu}\left(q^{2}\right) & =i\left(\frac{g v}{2 c_{W}}\right)^{2}\left[\Pi^{\prime}(0) g^{\mu \nu}+\Pi^{\prime \prime}\left(q^{2}\right) q^{\mu} q^{\nu}\right]
\end{aligned}
$$

where $v$ is the Higgs vev, and

$$
\Pi^{\prime}(x) \equiv \frac{\Pi(x)-\Pi(0)}{x}, \quad \Pi^{\prime \prime}(x) \equiv \frac{\Pi^{\prime}(x)-\Pi^{\prime}(0)}{x} .
$$

The values for the $W$ and $Z$ masses are shifted

$$
\begin{aligned}
& m_{W}^{2}=\left(\frac{g v}{2}\right)^{2}\left(1+\Pi^{\prime}(0)\right) \\
& m_{Z}^{2}=\left(\frac{g v}{2 c_{W}}\right)^{2}\left(1+\Pi^{\prime}(0)\right),
\end{aligned}
$$

as well as the propagators

$$
\frac{i}{q^{2}-m_{W, Z}^{2}}\left[-g^{\mu \nu}+\frac{\left(1+\frac{m_{W, Z}^{2} \Pi^{\prime \prime}\left(q^{2}\right)}{1+\Pi^{\prime}(0)}\right) q^{\mu} q^{\nu}}{m_{W, Z}^{2}+q^{2} \frac{m_{W, Z}^{2} \Pi^{\prime \prime}\left(q^{2}\right)}{1+\Pi^{\prime}(0)}}\right] .
$$

Let us first consider $f \bar{f} \rightarrow W_{L}^{+} W_{L}^{-}$in the EFT approach. The operator modifies the $H W^{+} W^{-}$and the $H f \bar{f}$ interactions. The combined effect is a factor of $1+\Pi^{\prime}(s)$. Therefore in this process the EFT approach is equivalent to the following substitution of the Higgs propagator:

$$
\Delta_{H}^{-1}(s)=\frac{1+\Pi^{\prime}(s)}{s-s_{H}+\Pi(s)},
$$

which behaves like $1 / s$ at large energy, and therefore exactly cancels the high-energy behavior from $\mathcal{M}_{t}^{L}$. It is also interesting to note that, if $\Pi(s)$ has a linear dependence on $s$, i.e.,

$$
\Pi(s)=i\left(s-\mu^{2}\right) \frac{\gamma}{\mu},
$$

the above equation becomes

$$
\Delta_{H}^{-1}(s)=\frac{1+i \frac{\gamma}{\mu}}{s-\mu^{2}+i s \frac{\gamma}{\mu}}
$$

and the EFT approach coincides with the scheme proposed by Seymour [13]. This makes sense because in the Seymour scheme the vector boson pair self energy also has a linear dependence on $s$. In our scheme we see that the numerator of Seymour's propagator comes from the modified $H W^{+} W^{-}$vertex, as required by the WI.

We now turn to vector-vector scattering and in particular to $W_{L}^{+} W_{L}^{-} \rightarrow Z_{L} Z_{L}$. This process features a pure gauge and a Higgs-mediated $s$-channel contribution, Fig. 2, Both contributions do contain terms that grow as $s$ at high energy, whose cancellation is guaranteed by gauge-invariance. To calculate $W_{L}^{+} W_{L}^{-} \rightarrow Z_{L} Z_{L}$ amplitude in the EFT we need to extract the Feynman rules from $\tilde{\mathcal{O}}_{\Pi}$, i.e., the contributions that need to be added to the usual SM rules. This is straightforward and gives (all momenta incoming):

$$
\begin{aligned}
& H(q) W^{+\mu}\left(k_{1}\right) W^{-\nu}\left(k_{2}\right) \\
& Z^{\mu}\left(k_{1}\right) W^{+\nu}\left(k_{2}\right) W^{-\rho}\left(k_{3}\right) \\
& Z^{\mu}\left(k_{1}\right) Z^{\nu}\left(k_{2}\right) W^{+\rho}\left(k_{3}\right) W^{-\sigma}\left(k_{4}\right) \\
& H \phi^{+} \phi^{-}, H \phi^{0} \phi^{0} \\
& \phi^{+} \phi^{-} \phi^{0} \phi^{0}
\end{aligned}
$$

$$
\begin{aligned}
& i g \frac{m_{W}}{\sqrt{1+\Pi^{\prime}(0)}} \Pi^{\prime}\left(q^{2}\right) g^{\mu \nu}+\cdots \\
& i \frac{g}{c_{W}} \frac{m_{W}^{2}}{1+\Pi^{\prime}(0)} s_{W}^{2}\left[\Pi^{\prime \prime}\left(k_{3}^{2}\right) g^{\mu \nu} k_{3}^{\rho}-\Pi^{\prime \prime}\left(k_{2}^{2}\right) g^{\mu \rho} k_{2}^{\nu}\right]+\cdots \\
& i g^{2} \frac{m_{Z}^{2}}{1+\Pi^{\prime}(0)}\left[\Pi^{\prime \prime}(s) g^{\mu \nu} g^{\rho \sigma}+s_{W}^{4}\left(\Pi^{\prime \prime}(t) g^{\mu \rho} g^{\nu \sigma}+\Pi^{\prime \prime}(u) g^{\mu \sigma} g^{\nu \rho}\right)\right]+\cdots \\
& -i \frac{g\left[m_{H}^{2}-\Pi(0)\right]}{2 m W} \sqrt{1+\Pi^{\prime}(0)} \\
& -i \frac{g^{2}\left[m_{H}^{2}-\Pi(0)\right]}{4 m_{W}^{2}}\left[1+\Pi^{\prime}(0)\right]
\end{aligned}
$$

where ellipsis denotes terms vanishing on shell and $s=\left(k_{1}+k_{2}\right)^{2}, t=\left(k_{1}+k_{3}\right)^{2}$, and $u=\left(k_{1}+k_{4}\right)^{2}$. These 
Feynman rules are sufficient to calculate both $W_{L}^{+} W_{L}^{-} \rightarrow$ $Z_{L} Z_{L}$ and $\phi^{+} \phi^{-} \rightarrow \phi^{0} \phi^{0}$. At the leading order in $\frac{m_{W}^{2}}{s}$ and $\frac{m_{W}^{2}}{m_{H}^{2}}$, we find for $W_{L}^{+} W_{L}^{-} \rightarrow Z_{L} Z_{L}$,

$$
\begin{aligned}
\mathcal{M}_{H}^{L L L L} & =-\frac{i g^{2}}{4 m_{W}^{2}} \frac{s^{2}\left[1+\Pi^{\prime}(s)\right]^{2}}{\left[s-m_{H}^{2}+\Pi(s)\right]\left[1+\Pi^{\prime}(0)\right]} \\
\mathcal{M}_{\text {gauge }}^{L L L L} & =\frac{i g^{2}}{4 m_{W}^{2}} s \frac{1+\Pi^{\prime}(s)}{1+\Pi^{\prime}(0)}
\end{aligned}
$$

and for $\phi^{+} \phi^{-} \rightarrow \phi^{0} \phi^{0}$,

$$
\mathcal{M}_{G}=-\frac{i g^{2}}{4 m_{W}^{2}} \frac{s+\Pi(s)-\Pi(0)}{s-m_{H}^{2}+\Pi(s)}\left[m_{H}^{2}-\Pi(0)\right]\left[1+\Pi^{\prime}(0)\right]
$$

so that

$$
\begin{aligned}
\mathcal{M}_{H}^{L L L L}+\mathcal{M}_{\text {gauge }}^{L L L L} & =-\frac{i g^{2}}{4 m_{W}^{2}} \frac{s+\Pi(s)-\Pi(0)}{s-m_{H}^{2}+\Pi(s)} \frac{m_{H}^{2}-\Pi(0)}{1+\Pi^{\prime}(0)} \\
& =\frac{\mathcal{M}_{G}}{\left[1+\Pi^{\prime}(0)\right]^{2}} .
\end{aligned}
$$

As expected, $\mathcal{M}_{H}^{L L L L}+\mathcal{M}_{\text {gauge }}^{L L L}$ does not grow with $s$ and the equivalence theorem is recovered, up to a factor $\left[1+\Pi^{\prime}(0)\right]^{2}$, which exactly amounts to the wave function renormalization of the Goldstone fields.

An interesting feature of our approach is that in the limit where the dependence of $\Pi(s)$ on $s$ is neglected, $\Pi(s) \equiv \Pi$ is a constant, then $\Pi^{\prime}(s)=\Pi^{\prime \prime}(s)=0$. The only effect of the operator is a shift in $\lambda$, the coupling of the Higgs-boson self interaction. If $m_{H}$ is the on-shell mass, this amounts to the replacement

$$
m_{H}^{2} \rightarrow m_{H}^{2}-\Pi,
$$

i.e., given that $\Pi$ can be a complex number, it is equivalent to the CMS.

The advantage of the EFT approach is the possibility of using "arbitrary" functional form of the self energy. We have shown that with special choices of $\Pi(s)$, the EFT approach can reduce to the Seymour scheme and the CMS scheme in certain cases. For example, there is no need for spurious non-zero width for $t$-channel propagators as this can be easily imposed by always maintaining gauge invariance. Finally, we note that despite the restoration of gauge-invariance and equivalence theorem is a general feature of our approach, one has to be careful in choosing the appropriate operator. For example, the following operator

$$
\mathcal{O}_{\Pi}^{\prime}=\frac{1}{2 v^{2}}\left(\phi^{\dagger} \phi-v^{2}\right) \Pi\left(-\partial^{2}\right)\left(\phi^{\dagger} \phi-v^{2}\right)
$$

introduced in Ref. 14], gives rise to the correct self energy and the resummed propagator, but it does not modify the gauge contribution, so in $W_{L}^{+} W_{L}^{-} \rightarrow Z_{L} Z_{L}$ the gauge cancellation between the $s$-channel Higgs-mediated amplitude and the gauge amplitude is not restored. On the other hand, it modifies the Goldstone amplitude in a way so that the equivalence theorem is satisfied. As a result, both $W_{L}^{+} W_{L}^{-} \rightarrow Z_{L} Z_{L}$ and $\phi^{+} \phi^{-} \rightarrow \phi^{0} \phi^{0}$ have bad high-energy behavior, and eventually break unitarity bounds. In general, adding higher dimensional operators to the Lagrangian leads to unitarity violation at some scale. We are going to show in the next sections that the operators we use do not have this problem.

Though the above operator $\mathcal{O}_{\Pi}^{\prime}$ solely does not treat the $H Z Z$ and $H W^{+} W^{-}$correctly at high energy, when combined with $\mathcal{O}_{\Pi}$, we can adjust them in a certain way to improve this method. We will discuss this in the following sections.

\section{UNITARITY}

Adding operators of dimension $n>4$ to the SM Lagrangian

$$
\mathcal{L}_{E F T}=\mathcal{L}_{S M}+\sum_{i} c_{i} \frac{\mathcal{O}_{i}[n]}{\Lambda^{n-4}},
$$

is equivalent to recast the SM in terms of an effective field theory valid up to scales of order $\Lambda$ [37], beyond which the theory is not unitary. It is therefore mandatory to check whether this is the case for the operator $\tilde{\mathcal{O}}_{\Pi}$. In fact, as we will see in the following section, a consistent perturbation theory implies that the same operator needs to also appear as a counterterm at higher orders. Overall we do not modify the theory and our procedure amounts to a reorganization of the perturbative expansion. However, we still need to make sure that neither unitarity is violated nor double counting happens at any given order in the perturbation theory. In this section we consider the first of these issues by showing that in sample calculations, $f \bar{f} \rightarrow V V$ and $V V \rightarrow V V$, at tree-level the operator in Eq. (19) does not break unitarity at large energy.

In $f_{ \pm} \bar{f}_{ \pm} \rightarrow V V$ the change in $H V V$ vertex cancels the change in $H$ propagator at high $s$, independently of the helicities of $V V$, so the $s$-channel Higgs diagram does not lead to any bad high-energy behavior. The scattering of opposite helicity fermions does not entail the $s$-channel Higgs diagram and is the same as in the SM.

As we have already verified, in $W^{+} W^{-} \rightarrow Z Z$ the longitudinal amplitude does not break unitarity, because the modification to the corresponding Goldstone interaction is finite $\left(m_{H}^{2}-\Pi(0)\right.$ and $\left.1+\Pi^{\prime}(0)\right)$. We now check the transverse amplitude $++\rightarrow--, 00 \rightarrow++,++\rightarrow 00$, $++\rightarrow++$, in the limit

$$
s \sim|t| \sim|u| \gg m_{W}^{2}, \quad m_{H}^{2} \gg m_{W}^{2} .
$$

(Note that $+-,+0,-0$ configurations do not feature a Higgs in the $s$-channel and therefore are left unchanged.) 
An explicit calculation for $W_{+}^{+} W_{+}^{-} \rightarrow Z_{-} Z_{-}$gives

$$
\begin{aligned}
& \mathcal{M}_{H}^{++--}=\mathcal{M}_{H}^{L L L L} \frac{4 m_{W}^{4}}{s^{2} c_{W}^{2}}+\mathcal{O}\left(m_{W}^{4}\right) \\
& \mathcal{M}_{\text {gauge }}^{++--}=\mathcal{M}_{\text {gauge }}^{L L L L} \frac{4 m_{W}^{4}}{s^{2} c_{W}^{2}}+\mathcal{O}\left(m_{W}^{4}\right),
\end{aligned}
$$

where $M^{L L L L}$ indicates the amplitude with four longitudinal vectors. For $W_{L}^{+} W_{L}^{-} \rightarrow Z_{+} Z_{+}$we obtain

$$
\begin{aligned}
& \mathcal{M}_{H}^{L L++}=\mathcal{M}_{H}^{L L L L} \frac{-2 m_{W}^{2}}{s c_{W}^{2}}+\mathcal{O}\left(m_{W}^{2}\right) \\
& \mathcal{M}_{\text {gauge }}^{L L++}=\mathcal{M}_{\text {gauge }}^{L L L L} \frac{-2 m_{W}^{2}}{s c_{W}^{2}}+\mathcal{O}\left(m_{W}^{2}\right),
\end{aligned}
$$

and for $W_{+}^{+} W_{+}^{-} \rightarrow Z_{L} Z_{L}$ we obtain

$$
\begin{aligned}
& \mathcal{M}_{H}^{++L L}=\mathcal{M}_{H}^{L L L L} \frac{-2 m_{W}^{2}}{s}+\mathcal{O}\left(m_{W}^{2}\right) \\
& \mathcal{M}_{\text {gauge }}^{++L L}=\mathcal{M}_{\text {gauge }}^{L L L L} \frac{-2 m_{W}^{2}}{s}+\mathcal{O}\left(m_{W}^{2}\right),
\end{aligned}
$$

These results vanish faster than the longitudinal amplitude at large $s$. Finally for $W_{+}^{+} W_{+}^{-} \rightarrow Z_{+} Z_{+}$, we obtain

$$
\begin{aligned}
\mathcal{M}_{H}^{++++} & =-i g^{2} m_{Z}^{2} \frac{\left[1+\Pi^{\prime}(s)\right]^{2}}{\left[s-m_{H}^{2}+\Pi(s)\right]\left[1+\Pi^{\prime}(0)\right]}+\mathcal{O}\left(m_{W}^{4}\right) \\
& \approx \frac{-i g^{2} m_{Z}^{2}}{1+\Pi^{\prime}(0)} \Pi^{\prime \prime}(s) \sim s^{-1} \quad \text { at large } s \\
\mathcal{M}_{\text {gauge }}^{++++} & =i 8 g^{2} c_{W}^{2} \frac{s^{2}}{4 t u}+\mathcal{O}\left(m_{W}^{2}\right)
\end{aligned}
$$

so at large energy the inclusion of $\tilde{\mathcal{O}}_{\Pi}$ does not lead to any bad high energy behavior.

\section{THE EFT APPROACH AT HIGHER ORDERS}

Starting at order $\alpha_{W}$, the operator $\tilde{\mathcal{O}}_{\Pi}$ is allowed in any leading order computation. At next-to-leading order in EW interactions, however, this is not necessarily consistent and possibly leads to double counting. In this section we argue that this is not a fundamental problem and can be dealt with by simply subtracting the same operator in a NLO as a counterterm, in full analogy to the procedure used in the CMS [8].

In the CMS, an imaginary part is added to the real mass, and then subtracted as counterterm at NLO. One can prove that this procedure does not spoil the WI's, despite the fact that only a special class of higher order terms is resummed. As the EFT approach can be viewed as a generalization to the CMS, the same approach can be followed. The operator $\tilde{\mathcal{O}}_{\Pi}$ corresponds to the imaginary part of the mass. It includes some of the higher-order contribution, and provides an improved solution to the
WI's. It enters the resummed propagator and other Feynman rules, and needs to be subtracted at higher orders. The main difference is that in the CMS the propagator describes an unstable particle with a fixed width, while in the EFT approach one can resum an arbitrary part of the self-energy correction. This difference may be important when the width of the unstable particle is large, as in the case of a heavy Higgs, and the actual functional form of $\Pi(s)$ becomes important.

In the pole-mass renormalization scheme, the twopoint function of the Higgs can be written as

$$
\Delta_{H}(s)=s-s_{H}+\Pi_{H H}^{R}(s)
$$

where $s_{H}$ is the pole and $\Pi_{H H}^{R}(s)$ is the one-loop PT selfenergy correction renormalized in the pole-mass scheme. We can now define the EFT approach by adding the operator in Eq. (19) and subtracting it as a counterterm:

$$
\mathcal{L}_{S M} \rightarrow \mathcal{L}_{S M}+\tilde{\mathcal{O}}_{\Pi}-\tilde{\mathcal{O}}_{\Pi} .
$$

In so doing the theory is exactly the same as before. Now Eq. (43) can be rewritten as

$$
\begin{aligned}
\Delta_{H}(s)= & s-s_{H}+\Pi(s) \\
& +\left[\Pi_{H H}^{R}(s)-\Pi(s)\right]
\end{aligned}
$$

where the first line starts at leading order, while the second line starts at order $\alpha_{W}$. The EFT approach then amounts to choose $\Pi(s)$ in a way to capture the important part of (if not all of) $\Pi_{H H}^{R}(s)$, so that this part of the self-energy correction is included at the leading order, and will be resummed. In practice, one does not have to choose the exact PT self energy, and gauge invariance is always guaranteed. In particular, choosing $\Pi(s)=0$ corresponds to the CMS scheme.

In our scheme, EW NLO calculations are obviously more involved. The resummed propagator (7) and the modified Feynman rules do require extra work. However, one can also always employ a standard CMS at NLO and only include the full propagators and vertices in the LO result. In this way we can consistently have leading order calculated in the EFT approach, and NLO in CMS but with counterterms from $\tilde{\mathcal{O}}_{\Pi}$.

\section{IMPROVED OPERATOR}

In the EFT approach, the exact functional form of the $\Pi(s)$ is somewhat arbitrary. Ideally, one would like to use the imaginary part of the PT one-loop self energy, as given in Eq. (9)-(12). Unfortunately, in $V V \rightarrow V V$ this choice produces an unphysical excess at low energy. As mentioned above, such an excess can be avoided by incorporating the operator $\mathcal{O}_{\Pi}^{\prime}$ in Eq. (32).

The reason for the excess is that the operator does not correctly describe the 1-loop $H V V$ vertices at low en- 
ergy. This can be traced back to the WI in Eq. $(15)^{3}$. The operator $\tilde{\mathcal{O}}_{\Pi}$ in Eq. (19) does not modify $\Gamma^{H \phi^{+} \phi^{-}}$, hence, the equality relation is completely satisfied by the $H W W$ vertex. At high energy this arrangement is satisfactory because we do not expect contributions from the Goldstone vertex. However, at low energy, this vertex turns out to be relevant and thus, modifying only the $H W W$ vertex gives rise to a bad behavior at low energy. On the other hand, the operator $\mathcal{O}^{\prime} \Pi$ (Eq. (32)) satisfies the equation with the saturation of the Goldstone-Higgs vertex, yet fails at describing the high energy behavior of weak boson scattering for example. The solution for this problem relies on the construction of a suitable combination of these two operators.

Let us rewrite them in the following form:

$$
\mathcal{O}_{\Pi_{1}}=\phi^{\dagger} \Pi_{1}\left(-D^{2}\right) \phi \text {. }
$$

Here we consider the operator of Eq. (19) in the simpler form, Eq. (17). This is justified by the fact that in practice we have to work only with the imaginary part of the self energy, then $\Pi(0)=0{ }^{4}$. On the other hand, the operator proposed by Beenakker et al. [14] (Eq. (32)), can be written as:

$$
\mathcal{O}_{\Pi_{2}}^{\prime}=\frac{1}{2 v^{2}}\left(\phi^{\dagger} \phi-v^{2}\right) \Pi_{2}\left(-\partial^{2}\right)\left(\phi^{\dagger} \phi-v^{2}\right) .
$$

To determine $\Pi_{1}$ and $\Pi_{2}$, we focus on the $H Z Z$ vertex and the $H \phi^{0} \phi^{0}$ vertex. The inclusion of $\mathcal{O}_{\Pi_{1}}$ and $\mathcal{O}_{\Pi_{2}}^{\prime}$ modifies these vertices, respectively:

$$
\begin{aligned}
k_{1}^{\mu} k_{2}^{\nu} \Gamma_{H Z Z, \mu \nu}\left(q, k_{1}, k_{2}\right) & =i \frac{g m_{Z}}{2 c_{W}} \Pi_{1}\left(q^{2}\right) \\
\Gamma_{H \phi^{0} \phi^{0}}\left(q, k_{1}, k_{2}\right) & =i \frac{g}{2 m_{W}} \Pi_{2}\left(q^{2}\right)
\end{aligned}
$$

where $k_{1}$ and $k_{2}$ are the momenta of $Z^{\mu} Z^{\nu}$ and $\phi^{0} \phi^{0}$. Now consider the following Ward Identity:

$$
\begin{aligned}
& k_{1}^{\mu} k_{2}^{\nu} \Gamma_{\mu \nu}^{H Z Z}\left(q, k_{1}, k_{2}\right)= \\
& -m_{Z}^{2} \Gamma^{H \phi^{0} \phi^{0}}\left(q, k_{1}, k_{2}\right)+\frac{i g m_{Z}}{2 c_{W}} \Delta_{H}\left(q^{2}\right) .
\end{aligned}
$$

This equation implies

$$
\Pi\left(q^{2}\right)=\Pi_{1}\left(q^{2}\right)+\Pi_{2}\left(q^{2}\right) .
$$

We now need to find out the expressions for $k_{1}^{\mu} k_{2}^{\nu} \Gamma_{H Z Z, \mu \nu}\left(q, k_{1}, k_{2}\right)$ and $\Gamma_{H \phi^{0} \phi^{0}}\left(q, k_{1}, k_{2}\right)$, or equivalently, $\Pi_{1}\left(q^{2}\right)$ and $\Pi_{2}\left(q^{2}\right)$. Then the combined operator $\mathcal{O}_{\Pi_{1}}+\mathcal{O}_{\Pi_{2}}^{\prime}$ should reproduce all three terms in Eq. (50) correctly. To this end, we calculate the absorptive part of both $k_{1}^{\mu} k_{2}^{\nu} \Gamma_{H Z Z, \mu \nu}\left(q, k_{1}, k_{2}\right)$ and $\Gamma_{H \phi^{0} \phi^{0}}\left(q, k_{1}, k_{2}\right)$ at one loop with PT. The results obtained are:

$$
\Pi_{1,2}(s)=\sum_{X X} \Pi_{1,2}^{X X}(s)
$$

where $X X$ are summed over $W W, Z Z, t t$ and $H H$, and 


$$
\begin{aligned}
\Pi_{1}^{W W}(s)= & -\frac{\alpha_{W}}{4 \pi}\left[2 s B_{0}\left(s, m_{W}^{2}, m_{W}^{2}\right)+\left(s-2 m_{Z}^{2}\right)\left(m_{H}^{2}+4 m_{W}^{2}\right) C_{0}\left(m_{Z}^{2}, m_{Z}^{2}, s, m_{W}^{2}, m_{W}^{2}, m_{W}^{2}\right)\right] \\
\Pi_{1}^{Z Z}(s)=- & \frac{\alpha_{W}}{16 \pi m_{W}^{2}}\left[\left(4 m_{Z}^{2} s+m_{H}^{4}+m_{H}^{2} m_{Z}^{2}-2 m_{Z}^{4}\right) B_{0}\left(s, m_{Z}^{2}, m_{Z}^{2}\right)\right. \\
& \left.+\left(4 m_{H}^{2} m_{Z}^{2} s+m_{H}^{6}-3 m_{H}^{2} m_{Z}^{4}-6 m_{Z}^{6}\right) C_{0}\left(m_{Z}^{2}, m_{Z}^{2}, s, m_{Z}^{2}, m_{H}^{2}, m_{Z}^{2}\right)\right] \\
\Pi_{1}^{t t}(s)= & \frac{3 \alpha_{W} m_{t}^{2}}{8 \pi m_{W}^{2}}\left[s B_{0}\left(s, m_{t}^{2}, m_{t}^{2}\right)+2 m_{t}^{2}\left(s-2 m_{Z}^{2}\right) C_{0}\left(m_{Z}^{2}, m_{Z}^{2}, s, m_{t}^{2}, m_{t}^{2}, m_{t}^{2}\right)\right] \\
\Pi_{1}^{H H}(s)= & \frac{3 \alpha_{W} m_{H}^{2}}{16 \pi m_{W}^{2}}\left[\left(m_{H}^{2}-m_{Z}^{2}\right) B_{0}\left(s, m_{H}^{2}, m_{H}^{2}\right)\right. \\
& \left.-\left(2 m_{Z}^{2} s+m_{H}^{4}-2 m_{H}^{2} m_{Z}^{2}-3 m_{Z}^{4}\right) C_{0}\left(m_{Z}^{2}, m_{Z}^{2}, s, m_{H}^{2}, m_{Z}^{2}, m_{H}^{2}\right)\right] \\
\Pi_{2}^{W W}(s)= & \frac{\alpha_{W}}{16 \pi m_{W}^{2}}\left[\left(m_{H}^{4}+4 m_{W}^{2} m_{H}^{2}+12 m_{W}^{4}\right) B_{0}\left(s, m_{W}^{2}, m_{W}^{2}\right)\right. \\
& \left.+4 m_{W}^{2}\left(s-2 m_{Z}^{2}\right)\left(m_{H}^{2}+4 m_{W}^{2}\right) C_{0}\left(m_{Z}^{2}, m_{Z}^{2}, s, m_{W}^{2}, m_{W}^{2}, m_{W}^{2}\right)\right] \\
\Pi_{2}^{Z Z}(s)= & \frac{\alpha_{W}}{32 \pi m_{W}^{2}}\left[\left(3 m_{H}^{4}+6 m_{H}^{2} m_{Z}^{2}+8 m_{Z}^{4}\right) B_{0}\left(s, m_{Z}^{2}, m_{Z}^{2}\right)\right. \\
& \left.+2\left(4 m_{H}^{2} m_{Z}^{2} s+m_{H}^{6}-3 m_{H}^{2} m_{Z}^{4}-6 m_{Z}^{6}\right) C_{0}\left(m_{Z}^{2}, m_{Z}^{2}, s, m_{Z}^{2}, m_{H}^{2}, m_{Z}^{2}\right)\right] \\
\Pi_{2}^{t t}(s)= & -\frac{3 \alpha_{W} m_{t}^{4}}{4 \pi m_{W}^{2}}\left[2 B_{0}\left(s, m_{t}^{2}, m_{t}^{2}\right)+\left(s-2 m_{Z}^{2}\right) C_{0}\left(s, m_{Z}^{2}, m_{Z}^{2}, m_{t}^{2}, m_{t}^{2}, m_{t}^{2}\right)\right] \\
\Pi_{2}^{H H}(s)= & \frac{3 \alpha_{W} m_{H}^{2}}{32 \pi m_{W}^{2}}\left[\left(m_{H}^{2}+2 m_{Z}^{2}\right) B_{0}\left(s, m_{H}^{2}, m_{H}^{2}\right)\right. \\
& \left.+2\left(2 m_{Z}^{2} s+m_{H}^{4}-2 m_{H}^{2} m_{Z}^{2}-3 m_{Z}^{4}\right) C_{0}\left(m_{Z}^{2}, m_{Z}^{2}, s, m_{H}^{2}, m_{Z}^{2}, m_{H}^{2}\right)\right]
\end{aligned}
$$

where $B_{0}$ and $C_{0}$ are the Passarino-Veltman functions [31]. Note in the above equations, only the imaginary part of both sides will be used.

One can verify explicitly that the WI in Eq. (50) is satisfied, i.e. with the above definition, we should have

$$
\Pi_{H H}^{(X X)}(s)=\Pi_{1}^{(X X)}(s)+\Pi_{2}^{(X X)}(s)
$$

for $(W W),(Z Z),(t t)$, and $(H H)$, respectively.

Note that $\Pi_{1}$ corresponds to $H Z Z$ and $\Pi_{2}$ corresponds to $H \phi^{0} \phi^{0}$. In Figure 3, we show a comparison of $\Pi_{H H}$, $\Pi_{1}$ and $\Pi_{2}$. At large energy, $\Pi_{H H}$ and $\Pi_{1}$ display the same behaviour, while $\Pi_{2}$ (i.e. $\left.H \phi^{0} \phi^{0}\right)$ is negligible. This justifies the use of our operator $\mathcal{O}_{\Pi}$ in Eq. (17) at high energy, as it generates the right $H V V$ vertex. At lower energy, however, $\Pi_{2}$ dominates and displays a "bump" above threshold. Because the operator given in Eq. (17) does not give rise to the right $H \phi^{0} \phi^{0}$ vertex, it is clear that had we chosen this operator in the EFT scheme, we would have mistakenly considered this "bump" as part of $H V V$ vertex, resulting in an unphysical excess at low energy.

To reproduce the right behaviour in both low and high energy regions we use the combined operator

$$
\overline{\mathcal{O}}_{\Pi}=\mathcal{O}_{\Pi_{1}}+\mathcal{O}_{\Pi_{2}}^{\prime}
$$

with $\Pi_{1}$ and $\Pi_{2}$ given by Eq. (52).
This operator gives a better description, as shown in Figure 4 where we compare the longitudinal component of the $H Z Z$ vertex derived from operator $\mathcal{O}_{\Pi}$ (Eq. (17)) and the improved operator $\overline{\mathcal{O}}_{\Pi}$ (Eq. (62) $)$ with the actual calculation at one loop with the PT. The improved operator describes the $H Z Z$ vertex very well. Figure 5 compares the transverse component of the $H Z Z$ vertex. The improved operator comes closer to the actual transverse component.

When applying our scheme with the operator $\overline{\mathcal{O}}_{\Pi}$ in Eq. (62), we prefer to work with the complex-pole renormalization. To do this we need the following counterterms for $\Pi_{H H}$ :

$$
\Pi_{H H}^{R}(s)=\Pi_{H H}(s)-\delta s_{H}+\left(s-s_{H}\right) \delta Z
$$

where $s_{H}$ is the pole. $\delta s_{H}$ and $\delta Z$ are given by

$$
\begin{aligned}
\delta s_{H} & =\Pi_{H H}\left(s_{H}\right) \\
\delta Z & =-\Pi_{H H}^{\prime}\left(s_{H}\right)
\end{aligned}
$$

For the Feynman rules, essentially we are going to replace all the $\Pi$ 's in (26) by $\Pi_{1}$, and include a factor of $1+\delta Z$ for the SM $H V V$ vertex to account for the wavefunction renormalization. 

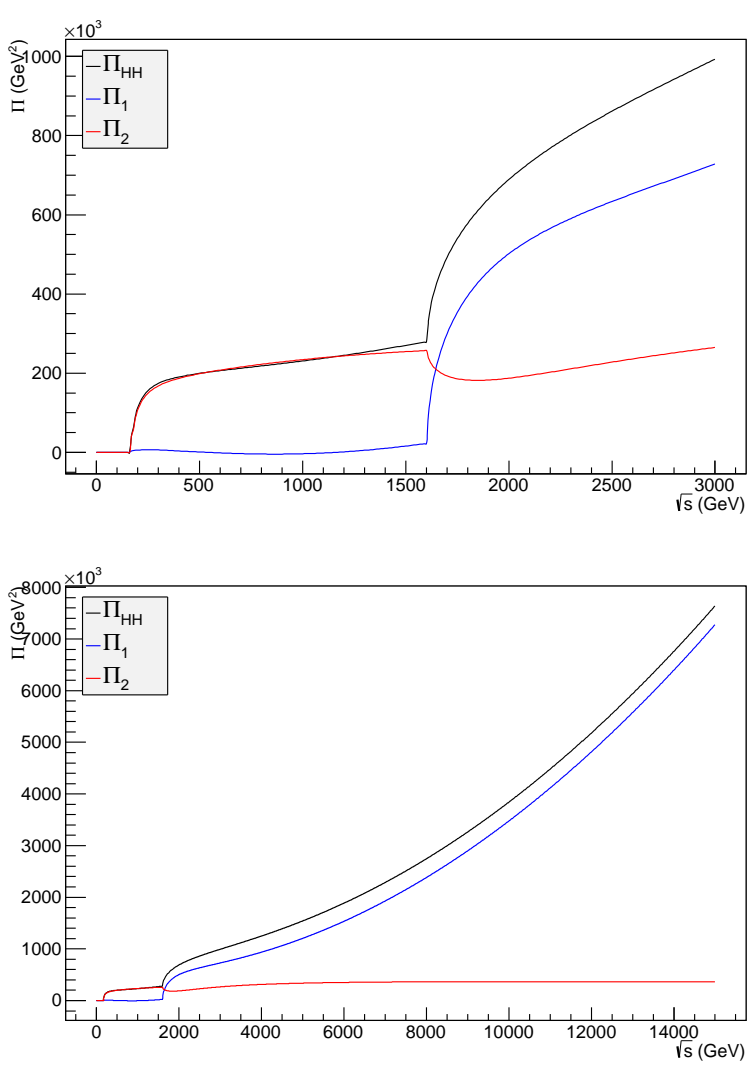

FIG. 3: Comparison of $\Pi_{H H}, \Pi_{1}$ and $\Pi_{2}$.

\section{APPLICATIONS}

The treatment of the propagator of the Higgs is of immediate relevance for the LHC. As simple testing ground of our proposal and comparisons to the conventional methods, we consider three processes of particular phenomenological importance at the LHC for a scalar boson (which for brevity, we identify with an hypothetical heavy Higgs): vector boson scattering, $t \bar{t}$ production via vector boson fusion and Higgs production via gluon fusion. We have compared the effective approach described above in Eqs. (21), (22) and (26), with two other schemes:

1. A naive inclusion of the self energy, i.e., using the following propagator

$$
\frac{i}{\Delta_{H}(s)}=\frac{i}{s-s_{H}+\Pi_{R}(s)},
$$

without changing anything else. Here $s_{H}=\mu^{2}-$ $i \mu \gamma$.

2. The CMS scheme,

$$
\frac{i}{\Delta_{H}(s)}=\frac{i}{s-s_{H}} .
$$
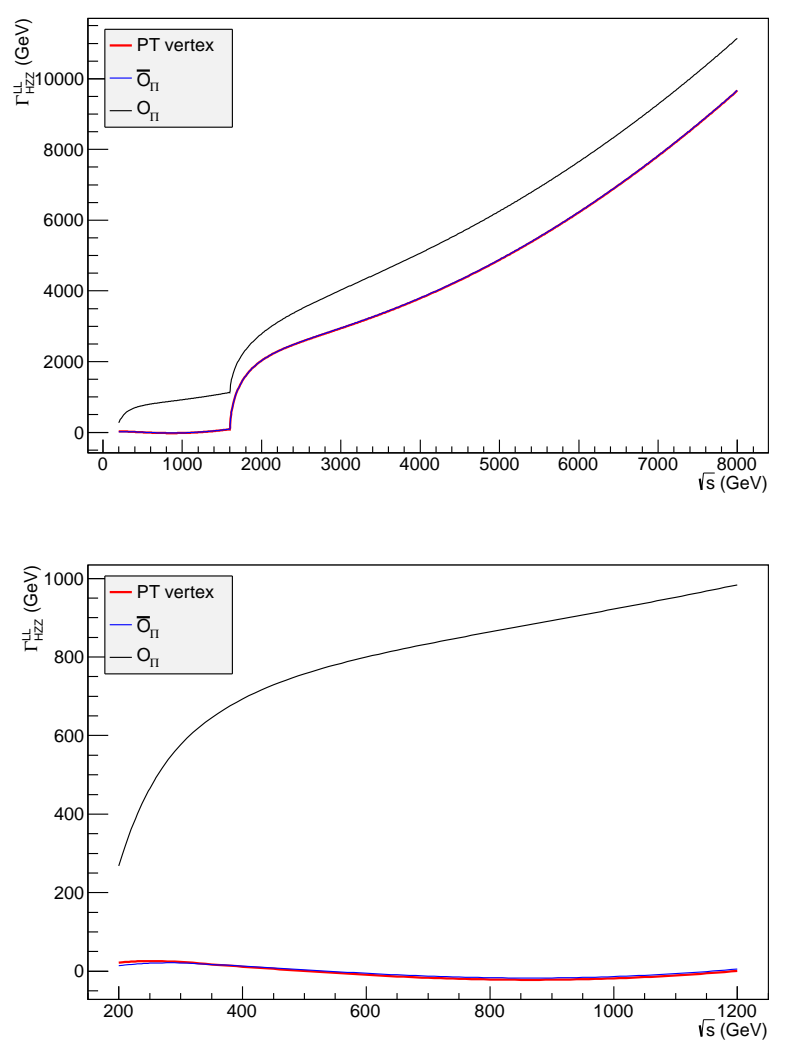

FIG. 4: The longitudinal components of the $H Z Z$ vertex.

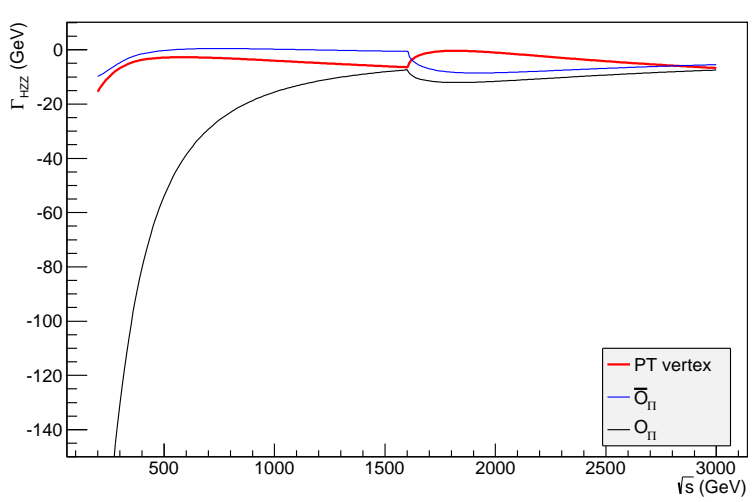

FIG. 5: The transverse components of the $H Z Z$ vertex.

A modified version of MADGRAPH 38, with the implementation of the effective Lagrangian approach and the naive propagator with the PT self energy is used to 
generate events. As SM input parameters we take:

$$
\begin{aligned}
& m_{Z}=91.188 \mathrm{GeV} \\
& G_{F}=1.16639 \times 10^{-5} \mathrm{GeV}^{-2} \\
& \alpha^{-1}=132.507 \\
& m_{t}=173 \mathrm{GeV} .
\end{aligned}
$$

The pole mass is

$$
\mu=800 \mathrm{GeV},
$$

and $\Pi_{R}(s)$ is the imaginary part of the PT self energy renormalized in the pole scheme. The factorization scale is set as the default dynamical scale of MADGRAPH and the PDF set is CTEQ611 [40].

\section{Vector Boson Scattering}

In $V V \rightarrow V V$ scattering processes, the effective description allows one to achieve a complete description of the Higgs line-shape at the resonance region and at the same time it corrects the bad high-energy behavior originated from the momenta dependent part of the self energy. As a bonus, we show that our definition avoids the need for including spurious $t$-channel widths which occur in the complex-mass scheme also affecting the high energy behavior of the scattering amplitudes.

In Figure [6] we show the energy behaviour of the $Z Z \rightarrow Z Z$ scattering amplitude summed over helicities, $\sum_{h e l}|M(s, t, u)|^{2}$, at scattering angle $\cos \theta=0$. The fixed width scheme, Eq. (67), naive propagator, Eq. (66), the effective description and a case in which the width is set to zero are presented. The agreement between the effective scheme and the naive propagator at the resonance region is pretty good. The difference with respect to the fixed width scheme is evident. At high energy, the naive propagator diverges, while the effective description behaves correctly. Similar comments can be made about $W^{+} W^{-} \rightarrow W^{+} W^{-}$amplitude, shown in Figure 7 .

The fact that in both $Z Z \rightarrow Z Z$ and $W^{+} W^{-} \rightarrow$ $W^{+} W^{-}$the fixed width scheme differs from the effective approach at the high energy region indicates that the spurious $t$-channel width gives a non-negligible contribution. This fact can be verified by comparing the different schemes with the no-width case. Moreover, in the case of $W^{ \pm} W^{ \pm} \rightarrow W^{ \pm} W^{ \pm}$, shown in Figure 8, the effective description and naive propagator are equivalent to the no-width case and the excess observed in the amplitudes in the fixed width scheme comes from the spurious width in the $t$ and $u$-channels.

At the LHC, the differences shown above may become important for a broad resonance. Despite the fact that a light Higgs has been observed, there is still room for new heavy and eventually broad resonances, e.g. in scaled-up QCD or in 2HDM's. The $V V$ scattering are embedded in

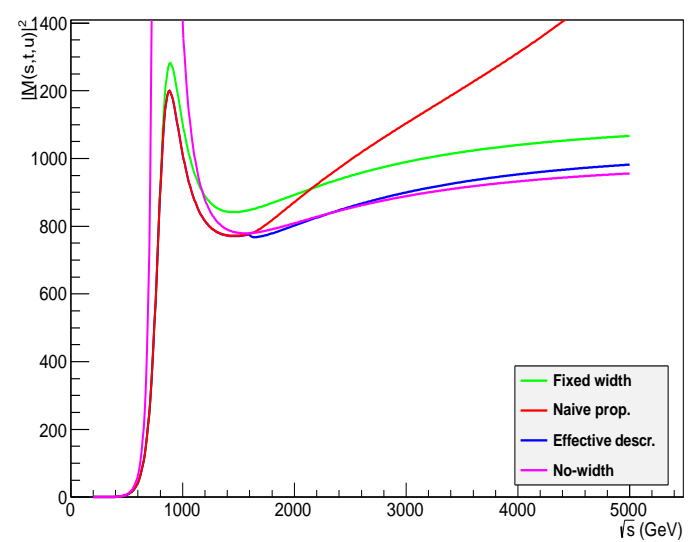

FIG. 6: $\sum_{\text {hel }}|M(Z Z \rightarrow Z Z)|^{2}$ with scattering angle, $\theta=$ $\pi / 2$. The curves correspond to: fixed width scheme, Eq. (67), naive propagator, Eq. (66), the effective description and the no-width, in which the width is set to zero.

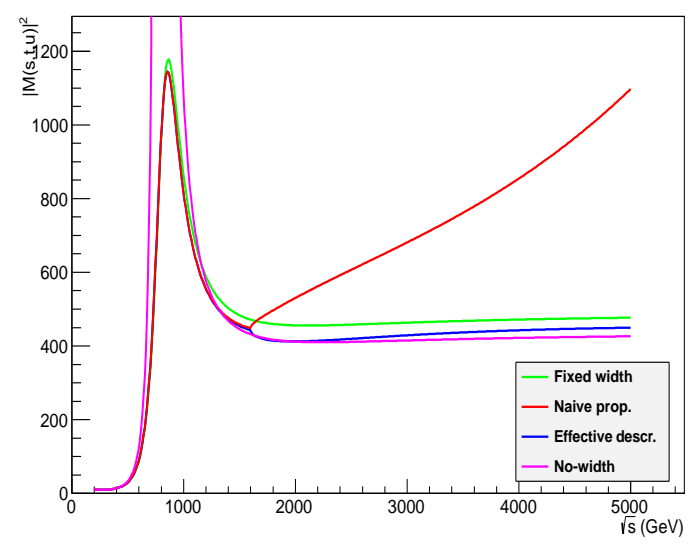

FIG. 7: $\sum_{h e l}\left|M\left(W^{+} W^{-} \rightarrow W^{+} W^{-}\right)\right|^{2}$ with scattering angle, $\theta=\pi / 2$. The curves correspond to: fixed width scheme, Eq. (67), naive propagator, Eq. (66), the effective description and the no-width, in which the width is set to zero.

more complex processes of the form $q q \rightarrow q q V V$, where the two final state jets are emitted with high energy in the forward-backward region of the detectors and the vector bosons decay into two fermions with high $p_{T}$ through the central region. We study the processes $u c \rightarrow u c Z Z$ and $u s \rightarrow d c W^{+} W^{-}$assuming the nominal energy of LHC, $E_{C M}=14 \mathrm{TeV}$.

In Figures 9, 10, the distribution of the invariant mass of the $Z Z$-system is shown. In Figure 9, the resonant region is shown. A basic set of selection cuts to enhance vector boson scattering contribution, listed in the left column of Table [. have been applied. The effective description fits well with the running behavior of the Higgs propagator. In Figure 10, the high-energy region is put in evidence. To better appreciate the differences 


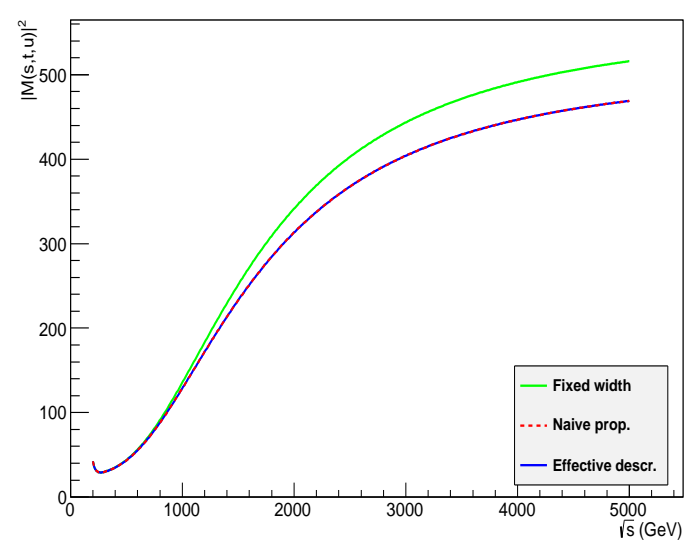

FIG. 8: $\sum_{\text {hel }}\left|M\left(W^{+} W^{+} \rightarrow W^{+} W^{+}\right)\right|^{2}$ with scattering angle, $\theta=\pi / 2$. The curves correspond to: fixed width scheme, Eq. (67), naive propagator, Eq. (66) and the effective description. The no-width case is equivalent to the last two.

between schemes at LHC energy, a further set of cuts has been added (right column of Table \). As expected, the effective approach gives a well behaved distribution at such energies contrary to the naive propagator and with a rate about $10 \%$ lower than the fixed width scheme. This difference amounts to the $t$-channel spurious contribution present in the fixed width case. Similar conclusions can be drawn from Figures 11, 12, where the reconstructed $W W$-system invariant mass distribution for the $u s \rightarrow d c W^{+} W^{-}$process is shown.

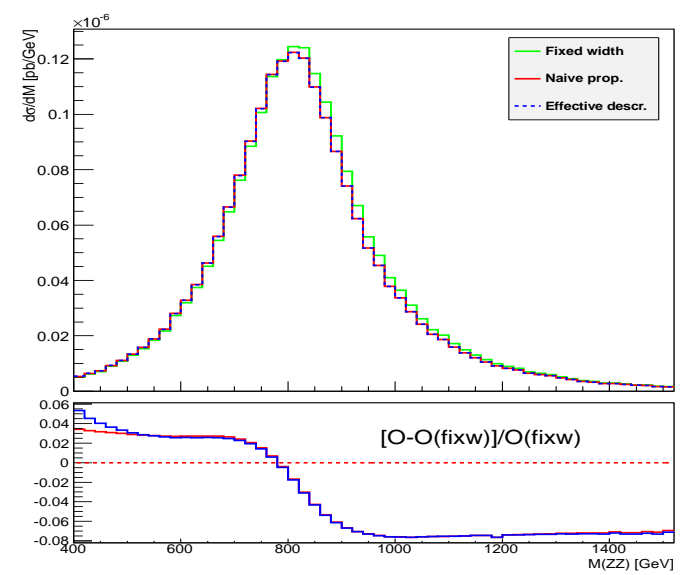

FIG. 9: Mass distribution of $Z Z$-system in the process $u c \rightarrow$ $u c Z Z$ around the resonance peak. The cuts listed in the left column of Table \have been applied.

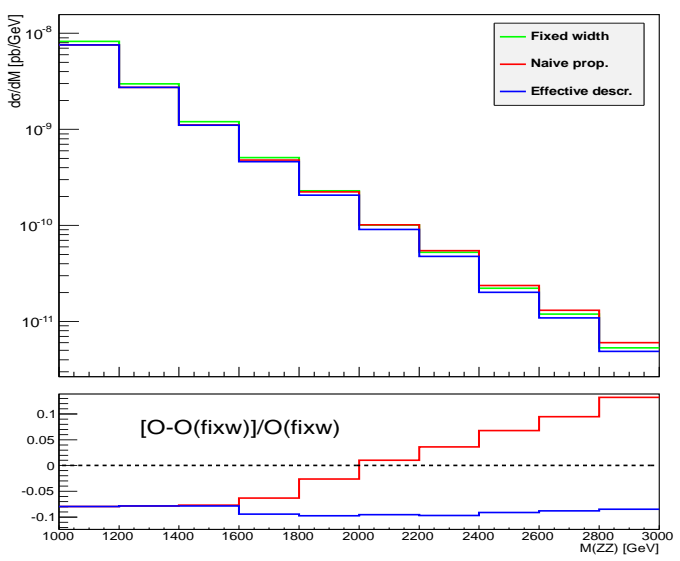

FIG. 10: Mass distribution of $Z Z$-system in the process $u c \rightarrow$ $u c Z Z$ at the high energy region. All cuts listed in Table $\prod$ have been applied.

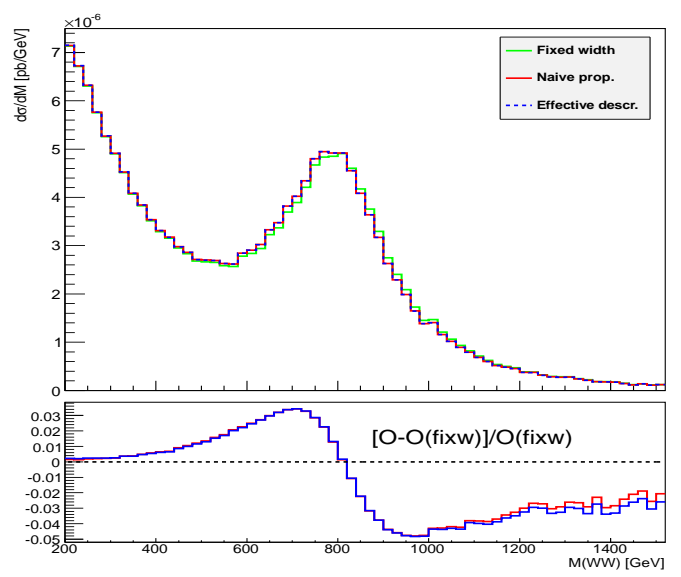

FIG. 11: Mass distribution of $W W$-system in the process $u s \rightarrow d c W^{+} W^{-}$around the resonance peak. The cuts listed in the left column of Table \ have been applied.

\begin{tabular}{|c|c|}
\hline Basic & Extra \\
\hline$p_{T}(j)>10 \mathrm{GeV}$ & $p_{T}(V)>400 \mathrm{GeV}$ \\
\hline $2<\eta(j)<10$ & $\Delta \eta(j j)>4.8$ \\
\hline$\Delta R(j, j)>4$ & $\eta(V)<2$ \\
\hline$M(j j)>100 \mathrm{GeV}$ & $M(j j)>1000 \mathrm{GeV}$ \\
\hline & $\Delta \eta(V, j)>1$ \\
\hline
\end{tabular}

TABLE I: Cuts to enhance vector boson fusion. Basic ones on the left column and the extra ones on the right column.

$$
W^{+} W^{+} \rightarrow H \rightarrow t \bar{t} \text { production }
$$

In $t \bar{t}$ production, we can observe a similar behavior with respect to $Z Z \rightarrow Z Z$ vector boson scattering. We have concentrated on the process $u s \rightarrow d c t \bar{t}$, in which the Higgs in produced by $W^{+} W^{-}$fusion and decayed to a 


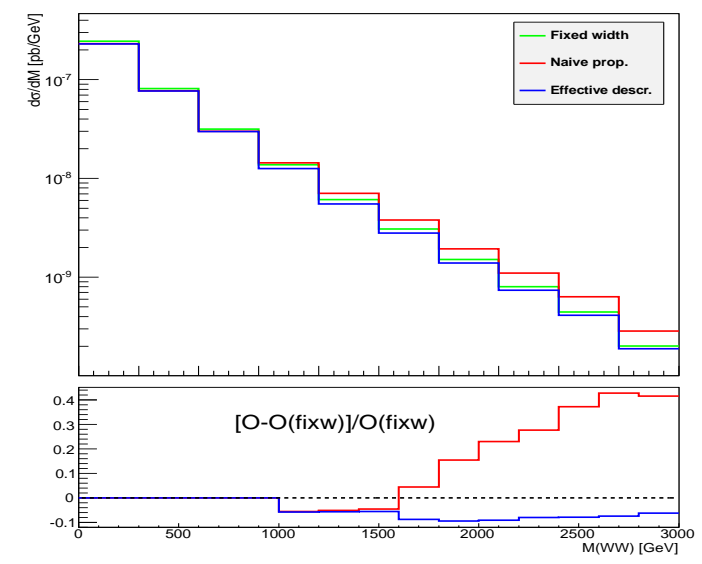

FIG. 12: Mass distribution of $W W$-system in the process $u s \rightarrow d c W^{+} W^{-}$at the high energy region. All cuts listed in Table \ have been applied.

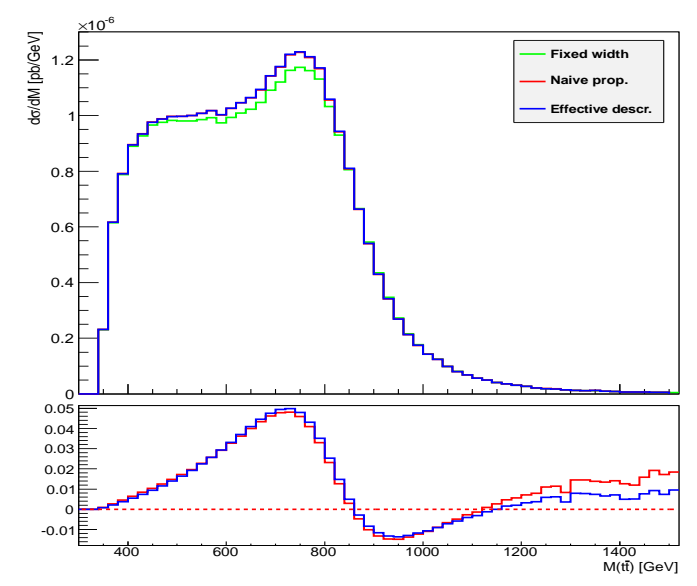

FIG. 13: Mass distribution of $t \bar{t}$-system in the process $u s \rightarrow$ $d c t \bar{t}$ around the resonance peak. The cuts listed in the left column of Table I have been applied.

pair of top quarks. The energy in the center of mass is set to $14 \mathrm{TeV}$. In Figure 13, the invariant mass distribution of $t \bar{t}$ at the resonant region is presented. The cuts shown in the left column of Table I have been applied in order to enhance the vector boson fusion contribution. Here again, the effective description describes the functional form of the propagator, which can go up to $5 \%$ of difference w.r.t the fixed width scheme. As seen in Figure 14, in the high mass region, the effective description is dumped down by the effective $W W H$ vertex and does not grow with energy as is the case in which the naive propagator is adopted. The extra cuts shown in the right-hand column of Table $\Pi$ have been added in order to highlight the differences better.

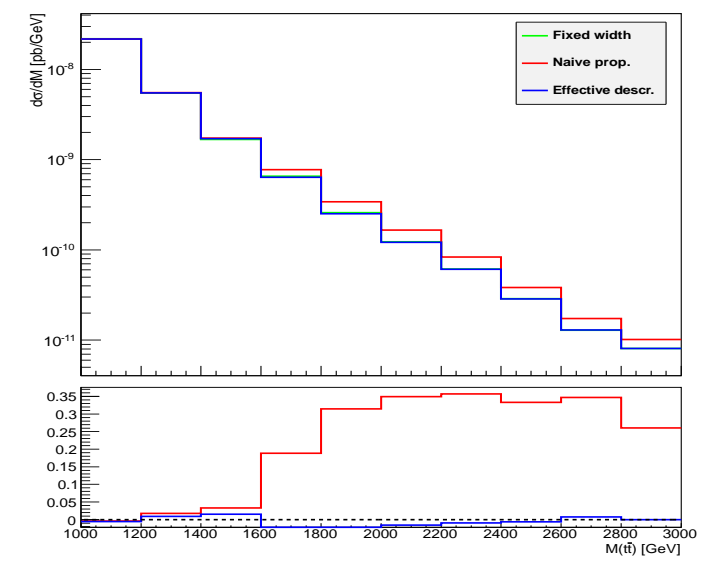

FIG. 14: Mass distribution of $t \bar{t}$-system in the process $u s \rightarrow$ $d c t \bar{t}$ at the high energy region. All cuts listed in Table $\$ have been applied.

\section{Gluon-gluon Fusion}

For the study of a heavy Higgs produced via gluongluon fusion and decayed to a $W$-boson pair, $g g \rightarrow$ $W^{+} W^{-}$, it is very important to consider the complete set of diagrams due to delicate gauge cancellations that control the high energy behavior. For this purpose we have relied on MCFM [39] for evaluation of the matrix elements, taking into account all diagrams contributing at leading order (yet one loop) to the process $g g \rightarrow W^{+} W^{-}$, with $W$ s decaying to leptons. Phase space integration and unweighted event generation have been carried out within the MADGraPh framework. The selection cuts shown in Table $\amalg$ have been applied.

$$
\begin{array}{|c|}
\hline p_{T}(\ell)>2 \mathrm{GeV} \\
\hline E_{T}>2 \mathrm{GeV} \\
\hline \eta(\ell)<3 \\
\hline \Delta R(\ell \ell)>0.5 \\
\hline
\end{array}
$$

TABLE II: Cuts applied for $g g \rightarrow W^{+} W^{-}$on the second one.

The mass distribution of $W W$-system in the three schemes considered if shown in Figure 15 and Figure 16. In Figure [15] we can see that the effective scheme and the naive propagator description present the same behavior around the resonance region, while the fixed width scheme shows a typical slighlity harder resonance. At high energy, the naive propagator diverges and the effective scheme and fixed width scheme are well behaved.

\section{CONCLUSIONS}

We have argued that it is possible to consistently and efficiently include running width effects for a heavy 


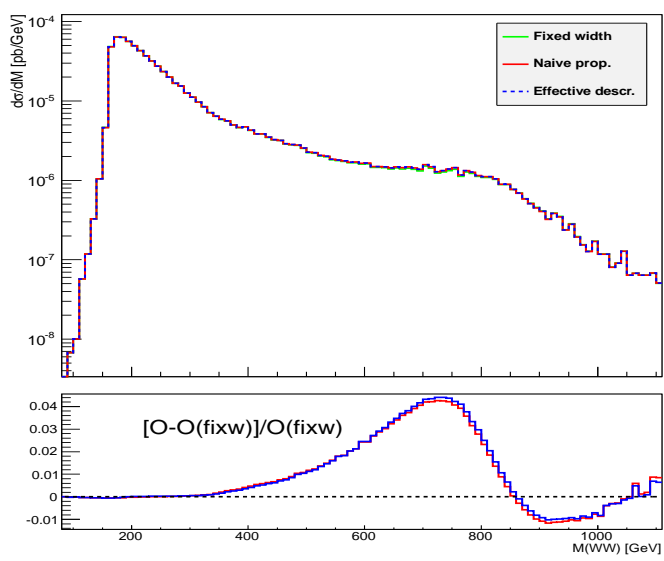

FIG. 15: Mass distribution of the reconstructed $W W$-system in the process $g g \rightarrow W^{+} W^{-} \rightarrow e^{+} \nu_{e} \mu^{-} \nu_{\mu}$, around the resonance peak. The cuts listed in Table【 have been applied.

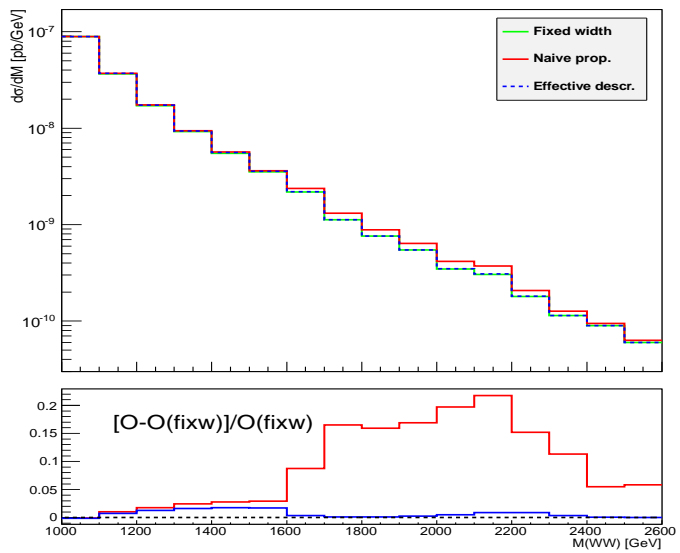

FIG. 16: Mass distribution of the reconstructed $W W$-system in the process $g g \rightarrow W^{+} W^{-}$at the high energy region. The cuts listed in Table $\amalg$ have been applied.

Higgs-like boson employing an EFT method. We can summarize the main points of our approach as follows:

- Introducing a width for an unstable particle amounts to a rearrangement of the perturbative expansion where the corrections to the two-point function are resummed in the propagator. The addition of the operator $\overline{\mathcal{O}}_{\Pi}$ defined in Eq. (62) allows to effectively perform such resummation in a gauge invariant and unitary way while keeping the full virtuality dependence of the self-energy. We have shown that in the limit where such dependence can be neglected our scheme is equivalent to the CMS.

- At leading order, one has the freedom to choose the functional form of $\Pi(s)$. We propose to use the exact one-loop PT self-energy correction. The rationale is that such self-energies are gauge invari- ant and by exploiting the WI's, we demand $\tilde{\mathcal{O}}_{\Pi}$ to mimic the most important one-loop corrections as much as possible. In practice, however, using any other form of $\Pi(s)$ does not break either gauge invariance or unitarity. In particular, one could avoid the need for a spurious non-zero width for $t$-channel propagators.

- EW higher-order corrections can be still performed in the CMS, without loss of accuracy or double counting issues. In practice, one can include the running width effects via the EFT at the leading order and neglect the virtuality dependence at NLO, i.e., employ the usual CMS for the NLO term.

In conclusion, in this work we have considered the case of how to consistently define a running width in the case of a heavy SM Higgs. The same approach can be used, for example, in the context of a Two-Higgs-Doublet model and applied to the current searches for new scalar states at the LHC. Extension to gauge vectors and heavy fermion states, on the other hand, are not straightforward and need further investigation.

\section{ACKNOWLEDGEMENTS}

We would like to thank Giampiero Passarino for many discussions, patient explanations, and comments on the manuscript. We are grateful to Vincent Mathieu for helpful discussion on the pinch technique. The work of D.B.F has been supported by an FSR incoming post-doctoral fellowship co-funded by the Marie Curie actions of the European Commission under contract SPER/DST/2661118906 , by the BELSPO (by the IAP Program, BELSPO VII/37, the IISN-FNRS convention 4.4511.10 "Fundamental interactions").

[1] G. Aad et al. [ATLAS Collaboration], arXiv:1207.0319 [hep-ex]; [CMS Collaboration], CMS-PAS-HIG-12-020.

[2] S. Chatrchyan et al. [CMS Collaboration], Phys. Lett. B 716, 30 (2012) arXiv:1207.7235 [hep-ex]].

[3] N. Kauer and G. Passarino, JHEP 1208 (2012) 116 arXiv:1206.4803 [hep-ph]].

[4] G. Passarino, JHEP 1208, 146 (2012) arXiv:1206.3824 [hep-ph]].

[5] S. Goria, G. Passarino and D. Rosco, Nucl. Phys. B 864, 530 (2012) arXiv:1112.5517 [hep-ph]].

[6] E. N. Argyres, W. Beenakker, G. J. van Oldenborgh, A. Denner, S. Dittmaier, J. Hoogland, R. Kleiss and C. G. Papadopoulos et al., Phys. Lett. B 358, 339 (1995) hep-ph/9507216.

[7] A. Denner, S. Dittmaier, M. Roth and D. Wackeroth, Nucl. Phys. B 560, 33 (1999) hep-ph/9904472.

[8] A. Denner, S. Dittmaier, M. Roth and L. H. Wieders, Nucl. Phys. B $\mathbf{7 2 4}$ (2005) 247 [Erratum-ibid. B 
854 (2012) 504] hep-ph/0505042; A. Denner and S. Dittmaier, Nucl. Phys. Proc. Suppl. 160 (2006) 22 hep-ph/0605312.

[9] S. Actis, G. Passarino, C. Sturm and S. Uccirati, Phys. Lett. B 669, 62 (2008) arXiv:0809.1302 [hep-ph]]; S. Actis, G. Passarino, C. Sturm and S. Uccirati, Nucl. Phys. B 811, 182 (2009) arXiv:0809.3667 [hep-ph]]; G. Passarino, C. Sturm and S. Uccirati, Nucl. Phys. B 834, 77 (2010) arXiv:1001.3360 [hep-ph]].

[10] W. Beenakker, G. J. van Oldenborgh, A. Denner, S. Dittmaier, J. Hoogland, R. Kleiss, C. G. Papadopoulos and G. Passarino, Nucl. Phys. B 500 (1997) 255 hep-ph/9612260.

[11] L. F. Abbott, Nucl. Phys. B 185 (1981) 189; L. F. Abbott, M. T. Grisaru and R. K. Schaefer, Nucl. Phys. B 229 (1983) 372; A. Denner, G. Weiglein and S. Dittmaier, Nucl. Phys. B 440 (1995) 95 hep-ph/9410338; A. Denner and S. Dittmaier, Phys. Rev. D 54 (1996) 4499 hep-ph/9603341.

[12] R. G. Stuart, Phys. Lett. B 262 (1991) 113; Phys. Rev. Lett. 70 (1993) 3193; A. Aeppli, G. J. van Oldenborgh and D. Wyler, Nucl. Phys. B 428 (1994) 126 hep-ph/9312212 ; H. G. J. Veltman, Z. Phys. C 62 (1994) 35.

[13] M. H. Seymour, Phys. Lett. B 354 (1995) 409 hep-ph/9505211.

[14] W. Beenakker, F. A. Berends and A. P. Chapovsky, Nucl. Phys. B 573 (2000) 503 hep-ph/9909472.

[15] W. Beenakker, A. P. Chapovsky, AKanaki, C. G. Papadopoulos and R. Pittau, Nucl. Phys. B 667 (2003) 359 hep-ph/0303105.

[16] M. Beneke, A. P. Chapovsky, A. Signer and G. Zanderighi, Phys. Rev. Lett. 93 (2004) 011602 hep-ph/0312331; M. Beneke, A. P. Chapovsky, A. Signer and G. Zanderighi, Nucl. Phys. B 686 (2004) 205 hep-ph/0401002.

[17] G. Valencia and S. Willenbrock, Phys. Rev. D 42 (1990) 853; G. Valencia and S. Willenbrock, Phys. Rev. D 46 (1992) 2247.

[18] A. Sirlin, Phys. Lett. B 267 (1991) 240; S. Willenbrock and G. Valencia, Phys. Lett. B 259 (1991) 373; M. Passera and A. Sirlin, Phys. Rev. Lett. 77 (1996) 4146 hep-ph/9607253; B. A. Kniehl and A. Sirlin, Phys. Lett. B 440 (1998) 136 hep-ph/9807545; Phys. Rev. Lett. 81 (1998) 1373 hep-ph/9805390; F. Jegerlehner, M. Y. .Kalmykov and O. Veretin, Nucl. Phys. B 641 (2002) 285 hep-ph/0105304; Nucl. Phys. B 658 (2003)
49 hep-ph/0212319.

[19] P. Gambino and P. A. Grassi, Phys. Rev. D 62 (2000) 076002 hep-ph/9907254; P. A. Grassi, B. A. Kniehl and A. Sirlin, Phys. Rev. Lett. 86 (2001) 389 hep-th/0005149; P. A. Grassi, B. A. Kniehl and A. Sirlin, Phys. Rev. D 65 (2002) 085001 hep-ph/0109228.

[20] B. A. Kniehl, C. P. Palisoc and A. Sirlin, Phys. Rev. D 66 (2002) 057902 hep-ph/0205304.

[21] J. M. Cornwall, Phys. Rev. D 26 (1982) 1453.

[22] J. M. Cornwall and J. Papavassiliou, Phys. Rev. D 40 (1989) 3474.

[23] J. Papavassiliou, Phys. Rev. D 41 (1990) 3179.

[24] G. Degrassi and A. Sirlin, Phys. Rev. D 46 (1992) 3104.

[25] J. Papavassiliou and A. Pilaftsis, Phys. Rev. Lett. 75 (1995) 3060 hep-ph/9506417.

[26] J. Papavassiliou and A. Pilaftsis, Phys. Rev. D 54 (1996) 5315 hep-ph/9605385.

[27] J. Papavassiliou and A. Pilaftsis, Phys. Rev. D 53 (1996) 2128 hep-ph/9507246.

[28] J. Papavassiliou and A. Pilaftsis, Phys. Rev. Lett. 80 (1998) 2785 hep-ph/9710380.

[29] J. Papavassiliou, hep-ph/9905328

[30] J. Papavassiliou and A. Pilaftsis, Phys. Rev. D 58 (1998) 053002 hep-ph/9710426.

[31] G. 't Hooft and M. J. G. Veltman, Nucl. Phys. B 153 (1979) 365; G. Passarino and M. J. G. Veltman, Nucl. Phys. B 160 (1979) 151.

[32] A. Denner, G. Weiglein and S. Dittmaier, Phys. Lett. B 333 (1994) 420 hep-ph/9406204.

[33] S. Hashimoto, J. Kodaira, Y. Yasui and K. Sasaki, Phys. Rev. D 50 (1994) 7066 hep-ph/9406271.

[34] J. Papavassiliou, Phys. Rev. D 51 (1995) 856 hep-ph/9410385.

[35] J. Papavassiliou, E. de Rafael and N. J. Watson, Nucl. Phys. B 503 (1997) 79 hep-ph/9612237.

[36] B. Grinstein, D. O'Connell and M. B. Wise, Phys. Rev. D 77, 025012 (2008) arXiv:0704.1845 [hep-ph]].

[37] S. Weinberg, Physica A 96 (1979) 327.

[38] J. Alwall, M. Herquet, F. Maltoni, O. Mattelaer and T. Stelzer, JHEP 1106 (2011) 128 arXiv:1106.0522 [hep$\mathrm{ph}]$.

[39] J. Campbell, R. K. Ellis, C. Williams, MCFM - Monte Carlo for FeMtobarn processes, http://mcfm.fnal.gov/

[40] J. Pumplin, D. R. Stump, J. Huston, H. L. Lai, P. M. Nadolsky and W. K. Tung, JHEP 0207 (2002) 012 hep-ph/0201195. 